\title{
LETTER FROM THE EDITOR IN CHIEF
}

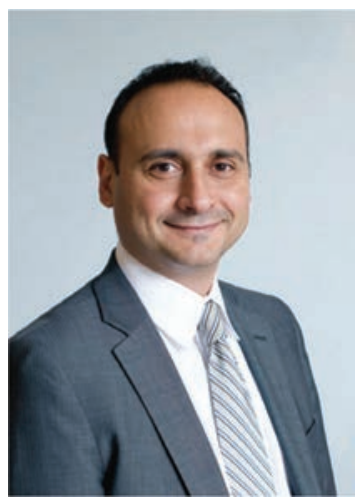

\author{
Dear Readers,
}

Transvenous pacemaker and ICD lead extraction is a very important component of the practice of cardiac electrophysiology. Over the past few years, there has been a strong interest in this topic. This increased awareness probably results from a rise in the rate of infection and recent recalls of ICD leads. It is critically important that every electrophysiologist caring for patients with pacemakers and ICDs to be knowledgeable with certain aspects of lead extraction, including indications for the procedure and complications associated with it, in order to make the correct referrals when faced with an infection or lead malfunction.

This issue of the Journal contains an important article related to lead extraction, entitled "Device Pocket Scar Predicts Transvenous Lead Extraction Difficulty". The authors describe the mechanisms of fibrosis and scar formation at points of contact between the lead and the cardiovascular structures. They demonstrated that the severity of scar and calcification in the device pocket correlates with the amount of scarring along the lead, a finding that may be used as a predictor for the degree of difficulty of extraction.

Detecting variables that predict the difficulty of the procedure is very important. Lead extraction can be associated with severe complications, including a mortality rate of $2.2 \%$ according to a recent report. The ability to predict the procedural challenges may allow the operator to prepare for difficult cases and perhaps reduce complications. Numerous publications have identified patient characteristics associated with increased mortality. These include age, female gender, systemic infection, local infection, device upgrade, high serum creatinine, and diabetes mellitus. However, predictors of endovascular scar formation have not been well described. Timely recognition of severe endovascular scarring may allow the operator to seek alternative approaches at an earlier stage of the procedure which may lead to improved outcome.

The identification of patients and procedural variables that can help predict the difficulty of the procedure is expected be more effective if combined with other practices that can ensure early detection and treatment of complications. A multidisciplinary approach including an electrophysiologist, an anesthesiologist with TEE expertise, and a standby cardiac surgeon, performing the extraction procedure in a hybrid operative room, is a promising model. The combination of detecting severe fibrosis early in the procedure and having the necessary resources available in the case of a perforation, is likely to improve the outcome associated with this complication

Best regards and I hope you enjoy reading this issue of the Journal which contains many important articles.

Regards,

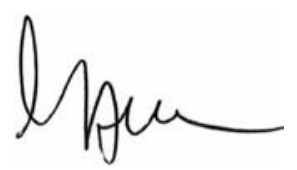

Moussa Mansour, MD, FHRS, FACC

Editor-in-Chief

The Journal of Innovations in Cardiac Rhythm Management

MMansour@InnovationsInCRM.com

Director, Cardiac Electrophysiology Laboratory

Director, Atrial Fibrillation Program

Massachusetts General Hospital

Boston, MA 